\title{
Improvement of Discrete Element Simulation Accuracy of Steel Powder Filling Behavior by Optimization of Contact and Friction Parameters
}

\author{
Naoki Yashiro ${ }^{1,2)}$, Kouya Oohira $^{2)}$, Natsuko Sugimura ${ }^{1)}$ and Hitoshi Washizu ${ }^{1,3)^{*}}$ \\ ${ }^{1)}$ Graduate School of Simulation Studies, University of Hyogo, 7-1-28 Minatojima-Minamimachi, Chuo-ku, Kobe, Hyogo 650-0047, Japan \\ ${ }^{2)}$ Advanced Technology R\&D Center, NTN Corporation, 5-105 Hidamarinooka, Kuwana, Mie 511-0867, Japan \\ ${ }^{3)}$ Elements Strategy Initiative for Catalysts and Batteries (ESICB), 1-30 Goryo-Ohara, Nishikyo-ku, Kyoto, Kyoto 615-8245, Japan
}

*Corresponding author: Hitoshi Washizu (h@washizu.org)

Manuscript received 10 September 2020; accepted 15 December 2020; published 31 January 2021

\begin{abstract}
The filling behavior of Fe-Ni-Mo steel powder for sintered machine parts is simulated using our original DEM (Discrete Element Method) code based on FDPS (Framework for Developing Particle Simulator). The difference between the simulated and experimental apparent densities after self-weight filling is only $-6.7 \%$, which is a significantly higher prediction accuracy than the prior study. This result is obtained by only calculating the repulsive force, viscous damping, friction force and gravity using the actual Young's modulus. This is due to using the experimental friction coefficients and the upper limit setting of the distance between the particle surfaces based on the experimental study. This method can be used to simulate the porosity, especially the low density region, in the actual filling process.
\end{abstract}

\section{Keywords}

steel powder, filling behavior, Hertzian contact, friction characteristics, Discrete Element Method (DEM), apparent density

\section{Introduction}

The sintered metal process is a manufacturing method that can produce complex-shaped machine parts, such as gears, sprockets, etc., from metal powders. The major advantage of this process is the near-net-shape manufacturing with a lower cost than that of the cutting of forged parts or castings. The replacement of cast steel parts with sintered steel ones is progressing, mainly for automobile engines. In recent years, in order to reduce the manufacturing costs, sintered parts with a higher strength or with more complicated fine shapes are desired [1-3]. For that purpose, it is required to achieve densification, reduction of pore size, and uniform filling of the mold. So far, application of the 2P2S (double pressing / double sintering) process [4], hot isostatic pressing (HIP) [5], or spark plasma sintering (SPS) [6], etc., has been proposed. However, these processes cannot utilize the advantages of sintered metal processes such as low manufacturing costs and near-net-shape manufacturing.

It is necessary to manufacture dense and strong machine parts via the conventional single pressing / single sintering (1P1S) process in order to gain a profit of the greatest advantage of sintered metals, which is the low manufacturing cost. Pores remaining inside of the sintered parts are inevitable, because the raw material is a metal powder. In addition, places where the powders are not densely packed become large pores, and sintered steels show a much lower densification during sintering than ceramics, so large pores do not disappear by sintering. Thus, steel powder has to be densely packed into the molds, but it is difficult to understand the powder characteristics inside the molds or to visualize the powders' behavior as well. It is important to develop a precise powder behavior prediction simulation.

Powder shows both fluid and solid behaviors, because it is influenced by its physical properties, particle density, external forces, etc. $[1,7,8]$. Thus, it is difficult to simulate its behavior by continuous methods, such as the finite element method (FEM) or by the finite difference method (FDM), which are generally used for the simulation of continuous body behavior. Powder behavior has been simulated by the discrete element method (DEM), which is one of the particle methods and requires no mesh formation. DEM is a method for analyzing the behavior of powder aggregates by tracking the behavior of individual particles, calculating the contact and non-contact interactions acting on the individual particles, and solving the equation of motion [9]. DEM, developed for geotechnical engineering, is a relatively simple method and a technique that can well describe macroscopic powder behavior, so it has been generally applied to the behavior analysis of powders and granular objects in the chemical engineering and pharmaceutical fields [10-12], and of 
course, sintered metal processes as well $[13,14]$

So far, in order to improve the simulation accuracy, many studies have been carried out, such as the proposals of new contact models $[15,16]$, verification and comparison of contact models or adhesion forces [17, 18], etc. However, it is known that the friction coefficients between particles and between particles and the mold are applied as empirical values or fitting parameters [13, 14], and there are few cases where the experimental values are applied [19]. In addition, in conventional DEM simulation studies, materials properties, such as Young's modulus and Poisson's ratio, have been treated as fitting parameters or empirical values, which are $10^{3}$ lower than the actual properties $[10,13,20]$. These simulation methods cannot treat the cases in which the raw material powder is replaced by another one with different material properties, in the actual manufacturing process development or material development.

In this study, in order to more precisely visualize and predict the steel powder behavior and to be used in manufacturing process development, we appllied the actual powder properties, such as Young's modulus, Poisson's ratio and friction coefficients, which are taken from experiments, to our original DEM code. Furthermore, several parameters related to the Hertzian contact calculation are optimized so as to fit the actual steel powder repulsion behavior. The DEM simulation accuracy is evaluated by the apparent density after self-weight filling, and is compared with the experimental value and that of a prior study [13].

\section{Methodology}

\subsection{Experimental}

The sample powder used in this study was the Fe-Ni-Mo steel powder with the particle size distribution from sub-micron to about $0.2 \mathrm{~mm}$, and $\mathrm{D}_{50}=0.1 \mathrm{~mm}$. The steel powder used in this study is a powder which is known as diffusion alloyed steel powder. The alloying elements, $\mathrm{Ni}$ and $\mathrm{Mo}$, are added to increase the hardenability, but they are very low (less than $5 \%$ in total). In addition, only Mo, which does not increase the hardness of the steel powder so much even after alloying, is pre-alloyed, while $\mathrm{Ni}$ is diffusely adhered to the steel powder surface as Ni particles. Therefore, mechanical properties such as Young's modulus and Poisson's ratio are similar to pure iron powder.

The powder characteristics were evaluated by the following two methods.

One is a conventional method for measuring the flowability and the apparent density using a flowmeter with a funnel, as standardized by JIS Z 2502 and 2504 (Fig. 1). The evaluation index in this study was the apparent density. The apparent density was evaluated by the filling fraction of the powder, which fell by its own weight from the funnel, into a cup with a $25 \mathrm{~cm}^{3}$ volume.

The other is a method using a powder yield locus (PYL), which is used in the field of powder engineering (Fig. 2). The fixed cell (the upper cell) is pushed against the powder layer filled by its own weight, and the powder layer (mobile cell) rotates while applying various normal loads. The evaluation indexes in this study were the inter-particle friction (internal friction angle) and friction between the particles and mold (wall friction angle). They were measured from the torque between the cell and the powder layer. A fixed cell with thin blades that causes shear inside of the powder layer was used when
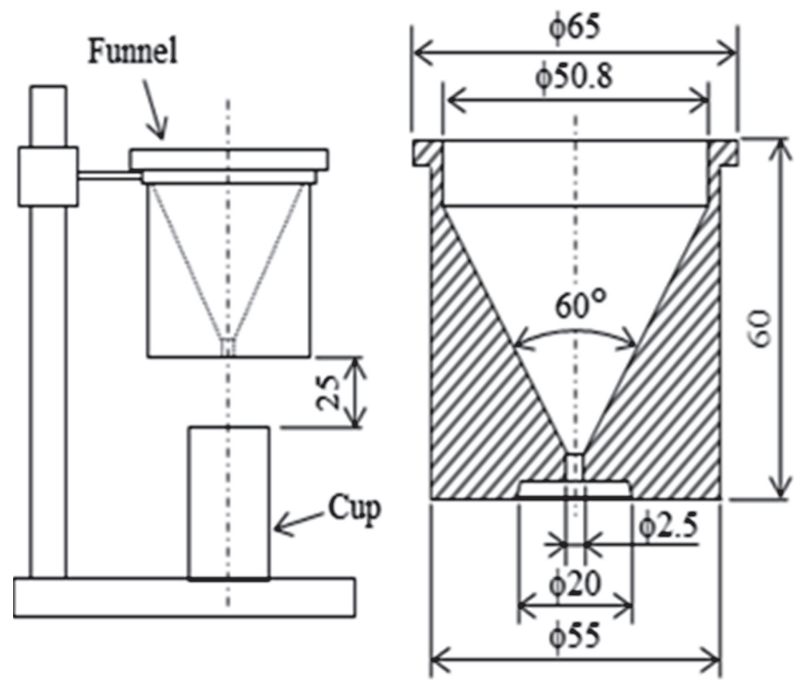

Fig. 1 Schematic of the funnel method. The left image is the appearance of the flowmeter consisting of a funnel and a cup. The right image is a cross section of the funnel, which satisfies the JIS Z 2502 standard. The unit of the values in the figure is $\mathrm{mm}$.

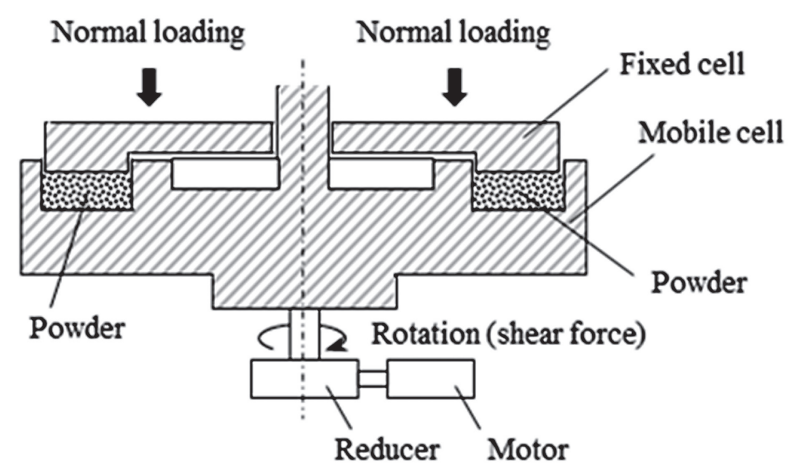

Fig. 2 Schematic of the PYL method. Fixed and mobile cells are ring shaped, and the sample powder is filled into the groove by its own weight. The powder layer is pushed against the upper fixed cell and rotates under various normal loadings. The friction indexes are calculated from the rotation torques between the fixed cell and powders.

measuring the internal friction angles, and a flat fixed cell that causes shear to the surface of the powder layer was used when measuring the wall friction angles. The lower these indexes, the better these frictions. By applying these friction angles $(\phi)$ to Eq. (1), the experimental friction coefficients $(\mu)$ were calculated.

$$
\mu=\tan \phi
$$

In addition, the repulsion behavior of the steel powder was experimentally evaluated, in order to optimize the parameters related to the contact force calculation in the DEM. The steel powder was dropped from the funnel shown in Fig. 1 onto the JIS SKD11 steel plate, which is the material generally used for the mold. The repulsion behavior of the steel powder was observed with a high speed microscope VW-9000 manufactured by Keyence Corporation. The experimental restitution coefficient was calculated using Eq. (2) from the height of before 
and after the repulsion $\left(h\right.$ and $\left.h^{\prime}\right)$.

$$
e=\frac{|v|}{\left|v_{0}\right|}=\frac{\sqrt{2 g h^{\prime}}}{\sqrt{2 g h}}=\sqrt{\frac{h^{\prime}}{h}}
$$

\subsection{Simulation}

We created the original DEM code which is implemented on FDPS (Framework for Developing Particle Simulator), with the view to developing into a large-scale parallelization in the future. FDPS is a particle method platform developed by Prof. Makino's group of RIKEN R-CCS [21]. FDPS is designed for improving the efficiency of the calculation loads and the load balances by employing a three-dimensional domain decomposition using the Multi-Section method [22], calculating long-range interactions using the Burnes-Hut algorithm, and calculating short-range interactions using the tree-search based neighbor list $[23,24]$. Thus, users only have to define the parameters of the particles and particle interactions, but do not have to program the codes related to the parallelization [25-29]. In this study, long-range interactions are not important, but the automatic domain decomposition is very important due to the discrete nature of the system.

The simulation conditions and the parameters are shown in Table 1. In this study, we created the model of spherical particles, which are assumed for the Fe-Ni-Mo steel powder as used in the experiments. They are regularly arranged on the top of the square prismatic mold and fall by their own weight. The mold has the size of $10 \times 10 \times 10 \mathrm{~mm}^{3}$, and is composed of spherical particles with the same size as the steel powder particles. The diameter of the particles is a uniform $0.3 \mathrm{~mm}$, which is the same as the prior study's model [13]. The number of particles is 15,028 for the powder and 7,344 for the mold, thus totaling 22,372. Although the actual steel powder has a particle size distribution as mentioned in Sect. 2.1., it is unified to the same particle size of $0.3 \mathrm{~mm}$ so that it can be compared with the prior study [13]. Further, although it is possible to introduce a histogram with several levels of particle size to approximate the actual particle size distribution, we have modeled it with a

Table 1 Coding and simulation conditions

\begin{tabular}{|c|c|c|c|}
\hline \multicolumn{2}{|c|}{ Parameter } & \multicolumn{2}{|l|}{ Value } \\
\hline Platform & & FDPS & \\
\hline \multirow[t]{5}{*}{ Particles } & Shape & Sphere & \\
\hline & Diameter $(d), \mathrm{m}$ & $3.0 \times 10^{-4}$ & \\
\hline & Number & (Powder) & 15,028 \\
\hline & & (Mold) & 7,344 \\
\hline & & (Total) & 22,372 \\
\hline \multirow{2}{*}{\multicolumn{2}{|c|}{ Contact model }} & \multirow{2}{*}{\multicolumn{2}{|c|}{$\begin{array}{l}\text { Non-linear spring model } \\
\text { (Hertzian contact) }\end{array}$}} \\
\hline & & & \\
\hline \multicolumn{2}{|c|}{ Young's modules, GPa } & \multicolumn{2}{|l|}{205} \\
\hline \multicolumn{2}{|c|}{ Poisson's ratio, - } & \multicolumn{2}{|l|}{0.27} \\
\hline \multicolumn{2}{|c|}{ Timestep, s } & \multicolumn{2}{|l|}{$1.0 \times 10^{-7}$} \\
\hline \multicolumn{2}{|c|}{ Number of steps } & \multicolumn{2}{|l|}{600,000} \\
\hline \multicolumn{2}{|c|}{ Simulated time, s } & \multicolumn{2}{|l|}{0.060} \\
\hline
\end{tabular}

single particle size in this study, in order to keep the calculation cost low.

We define that the steel powder particles move according to Newton's equation of motion [9], and employ the Leap-Frog method for time integration, which is often used in the field of DEM [30]. That is, Eqs. (3)-(5) have to be solved for the ensemble $i=1, \ldots ., N$ of $N$ particles, where $m_{i}$ is the mass, $\boldsymbol{r}_{i}$ is the center of the mass coordinate, $v_{i}$ is the velocity, $f_{i}$ is the total force of the $i$ th particle, and $\Delta t$ is the fixed timestep. The bold denotes a vector, and the dot denotes the first-order time derivative of each parameter. Although the self-weight filling of the steel powder into the mold is completed after $t=0.04$ to $0.05 \mathrm{~s}$, the calculation is performed until $t=0.06 \mathrm{~s}$, considering the time until the behavior of the steel powder is completely stabilized.

$$
\begin{aligned}
& m_{i} \dot{v}_{i}=f_{\mathrm{i}} \dot{r}_{\mathrm{i}}=v_{\mathrm{i}} \\
& r_{i}\left(t+\frac{1}{2} \Delta t\right)=\boldsymbol{r}_{i}\left(t-\frac{1}{2} \Delta t\right)+\boldsymbol{v}_{i}(t) \Delta t \\
& \boldsymbol{v}_{i}(t+\Delta t)=\boldsymbol{v}_{i}(t)+\frac{f_{t}(t) \Delta t}{m}
\end{aligned}
$$

In this study, we examine only the translational motion for the particle motion in order to verify how the simulation accuracy can be increased using as simple calculations as possible. We adopt a nonlinear spring model based on the Hertzian elastic contact theory for calculating the normal direction component of the inter-particle contact $[13,15,31$, 32]. It is modeled by a Voigt model consisting of a spring and a dash pod, and the repulsive force $f_{i j}^{e}$ is calculated by Eq. (6), and the viscous damping $f_{i j}^{v}$, which is defined by the Landau and Lifshitz model [33], is calculated by Eq. (7). The contact and the elastic deformation of two spherical particles $i$ and $j$ is described by the particles' radius and the distance of the $i-j$ particles' center. The parameter $h_{i j}=R_{i}+R_{j}-\left|r_{i j}\right|$ and $r_{i j}=r_{i}-r_{j}$, so $i-j$ particles contact at $h_{i j}=0$, and if $h_{i j}>0$, two particles elastically deform, as shown in Fig. 3, because all the particles in this study are rigid spheres with a single size. $\widetilde{E}=E /\left(1-v^{2}\right)$, where $E$ is Young's modulus and $v$ is Poisson's ratio. We apply the pure iron's actual physical constants, such as Young's modulus and Poisson's ratio, for the calculations, because the Fe-Ni-Mo steel powder for the sintered metal process is not hardened and shows the characteristics similar to pure iron as mentioned in Sect. 2.1. $R_{\text {eff }}$ is an effective particle radius and $R_{\text {eff }}=R_{i} R_{j} /\left(R_{i}+\right.$ $\left.R_{j}\right), \widehat{r_{i j}}$ is the normal unit vector of the contact plane, and $\gamma_{n}$ is an empirical damping parameter.

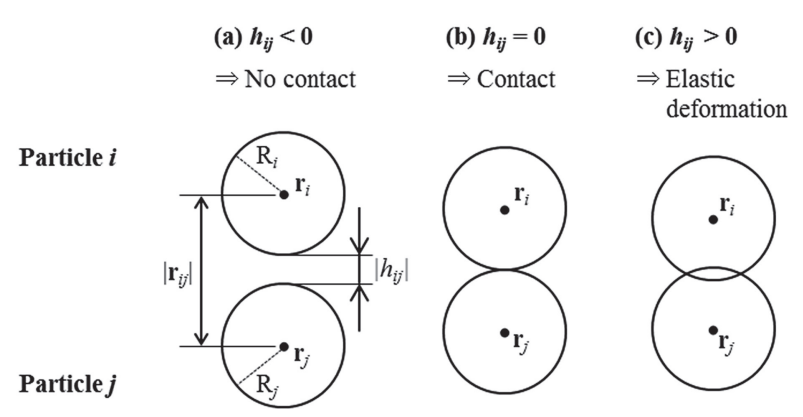

Fig. 3 Schematic of contact and elastic deformation of $i, j$ particles. (a) When $h_{i j}<0$, two particles don't contact, (b) when $h_{i j}=0$, contacting starts, and (c) when $h_{i j}>0$, contact and elastically deform. 


$$
\begin{aligned}
& f_{i j}^{e}=\left(\frac{2}{3} \tilde{E} \sqrt{R_{e f f}} h_{i j}^{2 / 3}\right) \widehat{r_{i j}} \\
& f_{i j}^{v}=-\left(\gamma_{n} \sqrt{R_{e f f} h_{i j}}\left(v_{i}-v_{j}\right) \cdot \widehat{r_{i j}}\right) \widehat{r_{i j}}
\end{aligned}
$$

In order to reduce the calculation load as much as possible, we adopt a tangential spring model based on Coulomb friction $[9,13]$, which is one of the most classic methods for the frictional force $f_{i j}^{t}$ calculation, as shown in Eq. (8), where $\kappa_{t}$ is a tangential spring constant, and $\xi_{i j}$ is elongation of the spring during contact. The friction force $f_{i j}^{t}$ is calculated by substituting the experimental friction coefficient obtained from Eq. (1) into the right side of Eq. (8).

$$
f_{i j}^{t}=-\min \left[\kappa_{t} \sqrt{\frac{h_{i j}}{R_{e f f}}}\left|\xi_{i j}\right|, \mu\left|f_{i j}^{e}+f_{i j}^{v}\right|\right] \frac{\xi_{i j}}{\left|\xi_{i j}\right|}
$$

In addition, for the sake of simplicity, only the gravity force $f_{i j}^{g}$ represented by Eq. (9) is applied as the non-contact force, where $g$ is the gravitational acceleration, and $\hat{z}$ is a unit vector in the z-direction. In this study, other effects, such as air resistance, adhesion force, and electrostatic force, are not considered for simplification.

$$
f_{i j}^{g}=-m_{i} g \hat{z}
$$

The accuracy of the DEM simulation in this study is verified by comparing the simulated apparent density values after self-weight filling with the experimental value and the prior study value [13]. We use the apparent density as an indicator to determine especially whether the sparseness of the particles could be evaluated quantitatively and whether the simulation could be used to predict the experiments. Since our model deals with more than 15,000 powder particles in a $10 \times 10 \times 10 \mathrm{~mm}^{3}$ cube, we think we can discuss the apparent density (filling fraction $=$ particle occupancy in the space) as an evaluation indicator. We optimize several parameters relating to the contact forces calculation by comparing the restitution coefficient between the experimental value and simulated one. Furthermore, we limit to some extent the displacement of $h_{i j}$ and $\left|\xi_{i j}\right|$ during the elastic contact. This is because it is experimentally known that the Hertzian elastic contact theory applied to the contact force calculation in this case is satisfied only in the region where the displacement due to contact is approximately $<20 \mathrm{~nm}$ for the iron-based material [34].

\section{Results and discussion}

\subsection{Experimental}

The apparent density of the steel powder due to self-weight filling was measured by the conventional funnel method, and found to be $3.00 \mathrm{~g} / \mathrm{cm}^{3}$. The indexes of the inter-particle friction and the friction between particles and mold, which cannot be evaluated by the funnel method, were measured by the PYL method. The former index is "internal friction angles" and the latter is "wall friction angles", and these indexes under various normal loads are shown in Fig. 4 . The lower the value, the lower the friction. The lowest normal load is a condition close to falling by its own weight, which is almost the same as the funnel method, and the higher load is one close to when the particles are elastically compacted and rearranged in the mold. In the DEM simulation of the powder filling or compaction molding process, it is necessary to input the friction coefficients between the powder and between the powder and mold. Thus, it is very meaningful that these friction indexes are experimentally obtained.

The experimental friction coefficients are easily calculated by applying the obtained friction angles to Eq. (1). The friction coefficients under the lowest normal load are shown in Table 2 as representative examples, since the self-weight filling behavior of the steel powder is going to be simulated by the DEM simulation. Each experimental friction coefficient value was found to be approximately two times higher than the value which was conventionally used in the DEM simulation [13, 14]. The friction coefficient of powder is difficult to measure stably, and number of prior DEM studies have used the friction coefficients as fitting parameters or empirical values. In particular, when the friction coefficient is used as a fitting parameter, it is optimized based on the results of contact force calculations using the unreal mechanical properties such as Young's modulus. Thus, it is not surprising that the friction coefficients in the prior studies are different from the

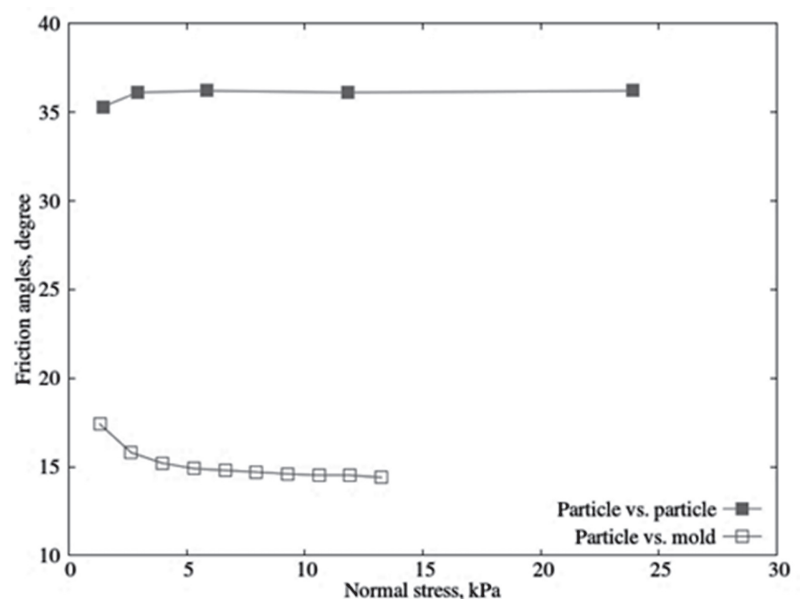

Fig. 4 Inter-particle and particle-mold friction indexes (friction angles) under various normal loads by the PYL method. The lower each index, the lower the friction.

Table 2 Experimental and prior study's friction coefficients. The friction coefficient of particle vs. particle is approximately 2.3 times higher than that of particle vs. mold, and the experimentally obtained values are approximately 2 to 2.4 times higher than those in the prior study [13], which was empirically used.

\begin{tabular}{ccccc}
\hline & $\begin{array}{c}\text { Friction angle } \phi, \\
\text { degree }\end{array}$ & Friction coefficient $\mu$, & $\mu$ in prior study [13], \\
& 35.3 & $\rightarrow$ & 0.71 & 0.30 \\
\hline Particle vs. particle & 17.4 & $\rightarrow$ & 0.31 & 0.15 \\
Particle vs. mold & & - & \\
\hline
\end{tabular}


experimental values. However, it is unclear why these values are less than half of the experimental values.

Furthermore, the restitution coefficient of the steel powder was calculated by Eq. (2) with the repulsion behavior when the powder fell onto the JIS SKD11 steel plate. The restitution coefficient of this steel powder due to self-weight falling onto the plate averaged 0.26 .

\subsection{Simulation}

The steel powder behavior when the powder fills the mold by its own weight is simulated using the FDPS based original DEM code with several parameter combinations. First, the combination of parameters, which relate to the contact force calculation represented by Eqs. (6)-(8), is optimized. In these calculations, there are two unknown parameters $-\gamma_{n}$ and $\kappa_{t}-$, which cannot be obtained by experiments and physical constants or calculated from the prior steps. In addition, $h_{i j}$ and $\xi_{i j}$ have to be verified in order to satisfy the Hertzian elastic contact theory and the appropriate powder behavior, as described in Sect 2.2. The fitted values of the various parameters related to the contact force calculations based on the restitution coefficient of the steel powder are shown in Table 3.

Table 3 Each parameters' optimal value in the contact forces calculation which is based on restitution coefficient data. $h_{i j}$ and $\left|\xi_{i j}\right|$ related to the calculations of the elastic contact forces are limited to the range, which satisfies the Hertzian elastic contact theory (Referring to prior study [34]).

\begin{tabular}{ll}
\hline Parameter & Value \\
\hline$R(=d / 2), \mathrm{m}$ & $1.5 \times 10^{-4}$ \\
$\gamma_{n}$, Pa s & $8.0 \times 10^{7} \times R$ \\
$\kappa_{t}$, Pa m & $5.0 \times 10^{7} \times R$ \\
The upper limit of $h_{i j}, \mathrm{~m}$ & $6.0 \times 10^{-7}$ \\
$\left|\xi_{i j}\right|, \mathrm{m}$ & $h_{i j} \times 600$ \\
\hline
\end{tabular}

In the prior study [13], the values of $\gamma_{n}$ and $\kappa_{t}$ are defined as the ratio to the particle radius $R$, and $\gamma_{n} / R$ and $\kappa_{t} / R$ are both $10^{6}$. In contrast, the optimal values of $\gamma_{n} / R$ and $\kappa_{t} / R$ in this study are $8.0 \times 10^{7}$ and $5.0 \times 10^{7}$, respectively, as shown in Table 3 , which is 80 or 50 times greater than the values in the prior study [13], respectively. The optimal values of the upper limit of the parameters related to the elastic contact $h_{i j}$ and $\left|\xi_{i j}\right|$ are $6.0 \times 10^{-7}$ and $h_{i j} \times 600$, respectively. The average restitution coefficient simulated with these parameters is 0.20 , which is suppressed to approximately $23 \%$ of the experimental value of 0.26 . In order to obtain the experimental parameters by a pure theoretical scheme, we need to complete the microscopic friction model [26-29].

Figure 5 shows the snapshots of before $(t=0 \mathrm{~s})$ and after $(t=$ $0.06 \mathrm{~s}$ ) the self-weight filling simulation of the steel powder. In this simulation, the experimentally obtained friction coefficients as shown in Table 2 are applied to Eq. (8). We now describe this condition as Case 1. The simulated apparent density of the selfweight-filled steel powder in Case 1 is compared to the result of the prior study [13] (Case 2) as shown in Table 4. The apparent density in Case 1 is obtained from the particle weight calculated from the number of particles existing in the lower region of the mold (for excluding the surface layer where the filled particles were not smooth) and the volume of the region. For the Case 2 simulation of the prior study, which is compared to Case 1 here, only the translational motion is considered, while rotation of the particles is not considered. They considered, however, cohesion in the contact forces calculation and air resistance in the non-contact forces calculation unlike our DEM code. The experimental apparent density of the steel powder used in this study is known to be $3.00 \mathrm{~g} / \mathrm{cm}^{3}$, as shown in Section 3.1 and Table 4. Compared to it, the simulated apparent density value of the prior study is $4.52 \mathrm{~g} / \mathrm{cm}^{3}$, that of this study is $2.80 \mathrm{~g} / \mathrm{cm}^{3}$, and the error versus the experimental value is $+50.7 \%$ and $-6.7 \%$, respectively. In other words, in the simulation of this study, the particles are slightly less packed than the actual steel powder, and in contrast, the particles are too densely packed than the actual powder in the prior study. The result in this study is more preferable in the powder filling simulation, because it is required to understand the places where the particles are not
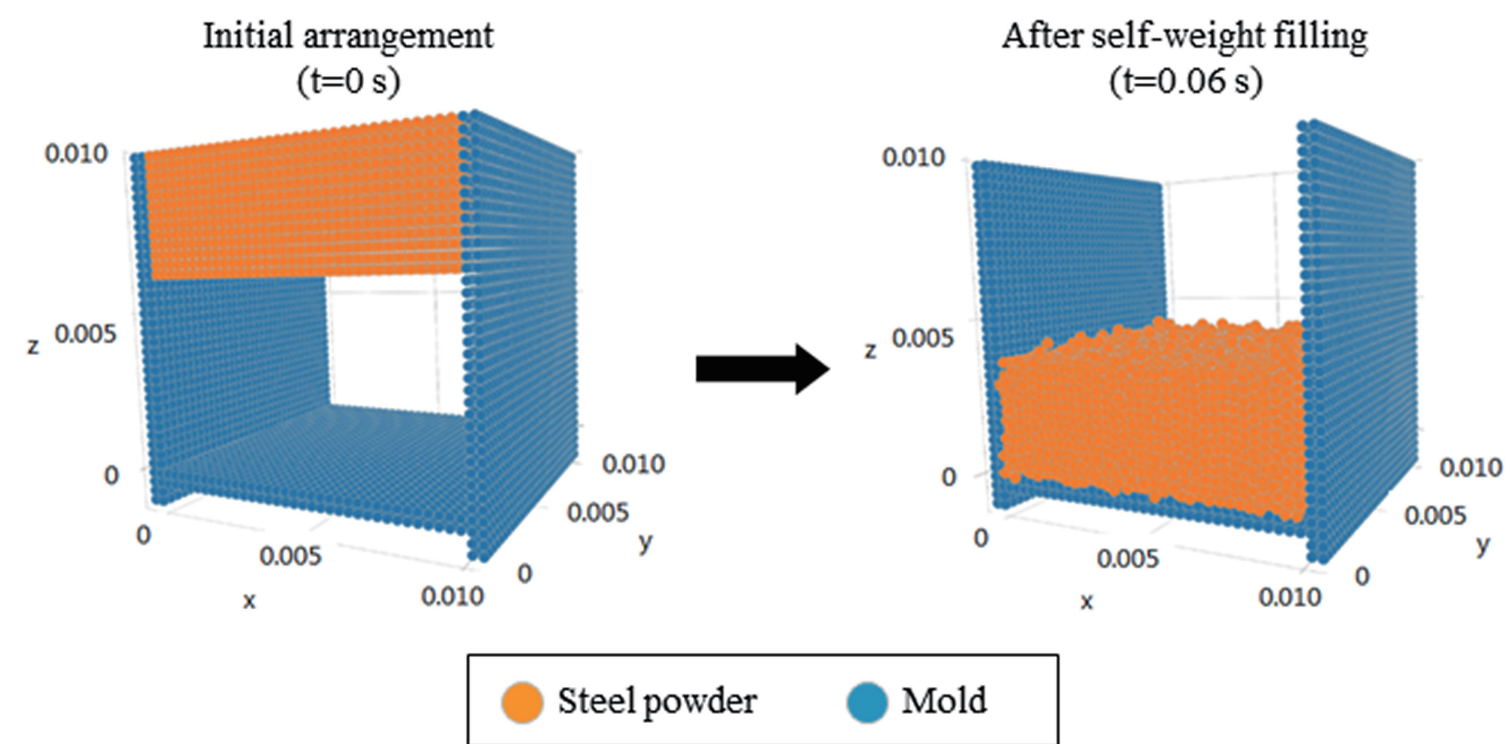

Fig. 5 The snapshots before and after the self-weight-filling simulation of the steel powder (Case 1). The orange spheres are the steel powder particles and the blue ones are the mold particles. The apparent density in Case 1 is $2.80 \mathrm{~g} / \mathrm{cm}^{3}$. 
Table 4 Simulated values of the apparent density. The apparent density in Case 1, which was calculated with our original DEM code and experimental friction coefficients, is $2.80 \mathrm{~g} / \mathrm{cm}^{3}$ and the difference vs. the experimental value is only $-6.7 \%$. The apparent density in Case 3, which was calculated with our original code and empirical friction coefficients, is much closer to that in Case 1 than that in Case 2.

\begin{tabular}{|c|c|c|c|c|c|c|}
\hline \multirow{2}{*}{ Case } & \multirow{2}{*}{ Simulation code } & \multirow{2}{*}{$\begin{array}{c}h_{i j,} \\
\times 10^{-7} \mathrm{~m} \\
\end{array}$} & \multicolumn{3}{|c|}{ Friction coefficient } & \multirow{2}{*}{$\begin{array}{c}\text { Apparent density, } \\
\mathrm{g} / \mathrm{cm}^{3}\end{array}$} \\
\hline & & & Type & Particle vs. particle & Particle vs. mold & \\
\hline 1 & This study & 6.0 & Experimental & 0.71 & 0.31 & 2.80 \\
\hline 2 & Prior study [13] & - & Empirical & 0.30 & 0.15 & 4.52 \\
\hline 3 & This study & 8.0 & $\uparrow$ & $\uparrow$ & $\uparrow$ & 3.08 \\
\hline Ref. & Experiment & - & - & - & - & 3.00 \\
\hline
\end{tabular}

densely packed.

In addition, the simulation accuracy is significantly improved compared to the prior study. In this study, the friction coefficients obtained from the experiment are applied, and the upper limit of the distance between the particle surfaces in the Hertzian contact calculation is restricted. Although it is considered that both are effective for improving the simulation accuracy, the following simulation (Case 3) is carried out in order to verify which one contributed more. Assuming the calculation conditions of the prior study [13] or the other general DEM simulation, an additional calculation is performed using our original DEM code. For the friction coefficients, the empirical values were applied instead of the experimental values, and the upper limit value of the inter-surface distance of the particles, $h_{i j}$, is raised to $8.0 \times 10^{-7}$, as shown in Table 4 . Figure 6 shows snapshots after the self-weight filling simulated under the conditions of Case 1 and Case 3. The shape of the powder layer is almost the same, and Case 3 (right) is more densely packed with the steel powder than Case 1 (left). In addition, some recoiled particles can be confirmed on the surface of the powder layer only in Case 3 . The simulated apparent density is $3.08 \mathrm{~g} / \mathrm{cm}^{3}$, which is closer to the result of Case $1(2.80 \mathrm{~g} /$

Case 1

$(\mathrm{t}=0.06 \mathrm{~s})$

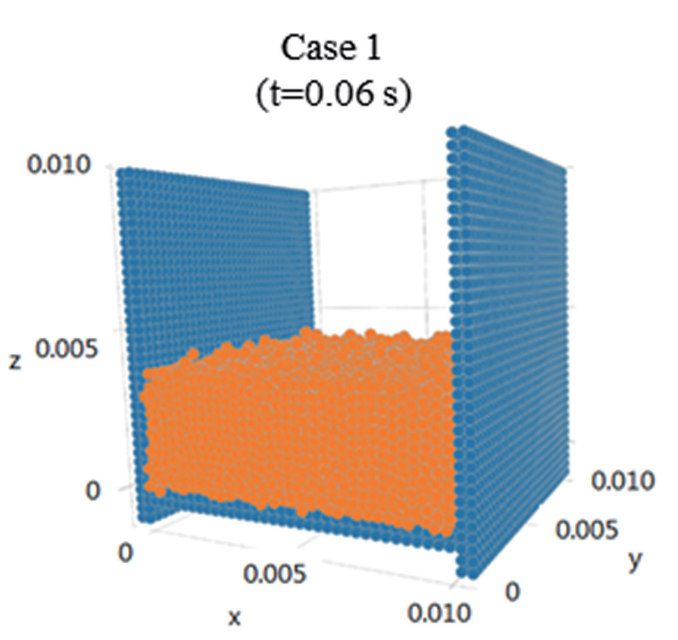

$\mathrm{cm}^{3}$ ) than that of Case $2\left(4.52 \mathrm{~g} / \mathrm{cm}^{3}\right)$. The apparent density in Case 3 is only 10\% higher than that in Case 1 in spite of the fact that the friction coefficients are more than $50 \%$ lower than in Case 1. In other words, it is considered that the setting of the upper limit of the distance between the particle surfaces in the Hertzian contact calculation contributes more to the natural powder behavior and the high accuracy of the packing density prediction in this study. It is considered that the powder behavior can be qualitatively and quantitatively realized by introducing this concept and optimizing parameters in spite of the fact that the actual Young's modulus of the raw material powder was applied.

The reasons why the simulated apparent densities in the prior study (Case 2) and in Case 3 excess the experimental value are considered to be as follows:

(1) Whereas the actual steel powder has complex shapes and rough surfaces, the prior study [13] and this study have modeled it with spherical particles.

(2) The prior study [13] used empirical low friction coefficients which are less than half of the experimental ones used in this study.

(3) The simulation model is composed of particles of $0.3 \mathrm{~mm}$

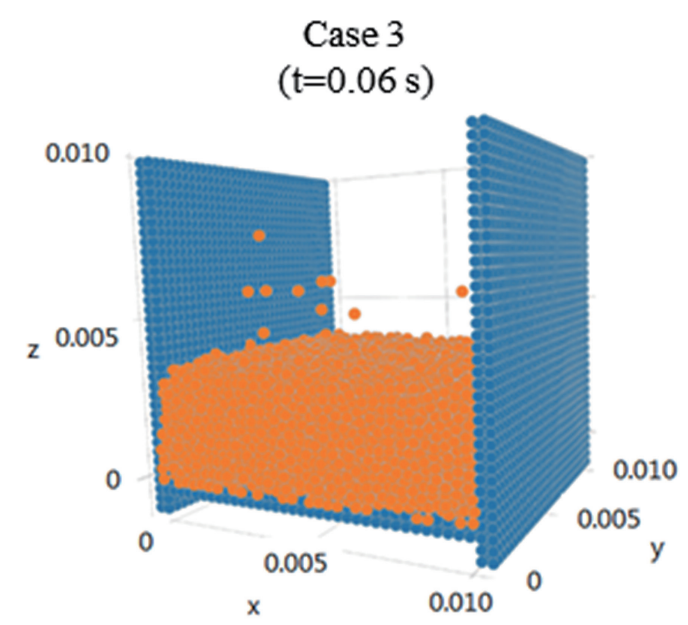

\section{Steel powder Mold}

Fig. 6 The snapshots after self-weight filling simulated under the conditions of Case 1 and Case 3. Case 3 (right) is more densely packed with steel powder than Case 1 (left). Furthermore, some recoiled particles can be confirmed on the surface of the powder layer in Case 3. 
larger than the actual particle size.

(4) Only the translational motion is considered for the particle motion but rotational motion.

The reason why the simulated apparent density in Case 2 is more than $50 \%$ higher than the experimental value would be mainly due to factors (1) and (2). In particular, for the factor (1), if the spherical particles are densely packed, the packing fraction can be 0.74 , as seen in the face-centered cubic (fcc) and hexagonal closest packing (hcp) in the crystal structure. Thus, since the true density of iron is approximately $7.8 \mathrm{~g} / \mathrm{cm}^{3}$, the apparent density can be $7.8 \times 0.74=5.772 \mathrm{~g} / \mathrm{cm}^{3}$. It is not that high since the friction coefficient, adhesion and etc. were taken into account in Case 2.

As for factor (2), the apparent density in Case 3, in which the friction coefficients are less than half of that in Case 1, is higher than that in Case 1 although the new concepts on contact force calculations are introduced in this study. Therefore, in case DEM simulation is used to discuss especially low density cases, it is important to use friction coefficients based on experiments.

The reason why the apparent density in Case 1 is lower than the experimental value would be due to factors (3) and (4). Spherical particles tend to have a higher packing density than actual complex shaped particles, as described above. In this study, however, the friction coefficients shown for actual complex shaped particles are applied to spherical particles in the simulation, so the apparent density is not excessively high as in the prior study. On the contrary, since the particles are larger in size than the actual particles, the voids between them are larger, and since only translational motion is considered, the particles are thought to be difficult to filling into the voids by rotation.

The reason why the simulation result of Case 3 is closer to the experimental value than that of Case 1 is that only the translational motion is considered in this study. When considering the rotational motion, it is considered that the packing property is further improved and the apparent density value is higher, so it will be close to the experimental value. The recoil particles were observed in Case 3, probably because the upper limit of $h_{i j}$, which is the distance between the particle surfaces, was raised. Thus, the particles in Case 3 can more strongly contact than in Case 1 resulting in an excessive repulsive force, $f_{i j}^{e}$.

In brief, the method used in this study can simulate the difference in the packing density by reflecting the difference in the friction coefficients. The friction coefficients can be experimentally obtained by the PYL method, as mentioned in the Sec. 3.1. Thus, in the actual development of powder materials or manufacturing processes, it is possible to more accurately simulate the powder behavior when the materials with different powder characteristics are used. Furthermore, in the prior study, as already described, a more rigorous interaction calculation is performed in consideration of the adhesive force and the air resistance. However, in this study, in spite of which only calculates the repulsive force, viscous damping, frictional force, and gravity, the simulation result with an apparent density that is much closer to the experimental value was obtained. Thus, it can be said that the knowledge based on the experiment, i.e., the friction coefficients and the setting of the upper limit of the distance between the particle surfaces, is very important and useful. In addition, since the calculation of the forces around the particles can be limited, it is considered that the calculation load can be reduced.

In this study, the simulation accuracy of the apparent density was significantly improved by reflecting the friction coefficient and the knowledge based on experiments in the contact force calculations. However, the particle size is three times larger than the actual $\mathrm{D}_{50}$ and the particle size distribution is not taken into account. Also, only translational motion of the particles is considered, not rotational motion. The addition of these items will lead to further improvement of the simulation accuracy, and they should be discussed in further research.

\section{Conclusion}

In this study, the filling behavior of the Fe-Ni-Mo steel powder for sintered machine parts was simulated using our original DEM code based on FDPS. The simulation accuracy was significantly improved mainly by the following two factors.

The first one is applying the experimental friction coefficients $\mu$ by the PYL method, which are 2 times or higher than the empirical $\mu$ s used in the conventional DEM.

The other one is setting of the upper limit of the distance between the particle surfaces, which is based on the experimental study that there is an upper limit of the indentation depth at which the Hertzian elastic contact theory holds.

Furthermore, by optimizing the parameters related to the contact forces calculations so that the simulated value of the restitution coefficient when dropping by its own weight was close to the experimental value, the error between the simulated and experimental apparent densities was only $6.7 \%$. In the prior study, the contact forces between particles are calculated using the friction coefficients, which are less than half the experimental value, and Young's modulus, which is 3 orders lower than the actual value, the simulated apparent density is $50.7 \%$ higher than the experimental value, despite consideration of the air resistance and adhesive force. It cannot be said that the porosity, especially the low density region, in the actual filling process can be evaluated by that simulation.

In fact, as a result of simulating under the conditions close to those of the prior study, the apparent density value increases, and recoil particles are also confirmed which is considered to be due to the excessive contact of the particles. In this study, only the repulsive force, viscous damping, frictional force and gravity are calculated, and the upper limit of the distance between the particle surfaces is set. Thus, it is possible to perform a highly accurate simulation with a lower calculation load, especially to evaluate the low density region.

\section{Acknowledgments}

The authors used a free platform "CedarPlot" for visualization of the simulated results. We would like to thank Dr. T. Sugimura of NiPR (National Institute of Polar Research), who is the developer of the platform. We would also like to thank Dr. Le Van Sang for his useful advice about the simulation using FDPS. This study was supported by JSPS KAKENHI Grant Numbers JP20K04245, JP18K18813, JP20H02058, and JP19H05718.

\section{References}

[1] German, R. M., "Powder Metallurgy and Particulate Materials Processing," Metal Powder Industries Federation, New Jersey, 2005.

[2] Tsutsui, T., "Recent Technology of Powder Metallurgy and Applications," Hitachi Chemical Technical Report, 54, 2012, 12-20. 
[3] Hanejko, F., "High Density via Single Pressing / Single Sintering," Powder Metallurgy Technology, 28, 1, 2010, 73-76.

[4] Azadbeh, M., Danninger, H. and Gierl-Mayer, Ch., "The Influence of First Compaction on Properties and Microstructure of Double Pressed Cr-Mo Prealloyed Sintered Steel," Powder Metallurgy Progress, 15, 1, 2015, 13-22.

[5] Das, S., Wohlert, M., Beaman, J. and Bourell, D., "Producing Metal Parts with Selective Laser Sintering/Hot Isostatic Pressing," Journal of the Minerals, Metals \& Materials Society, 50, 12, 1998, 17-20.

[6] Mamedov, V., "Spark Plasma Sintering as Advanced PM Sintering Method," Powder Metallurgy, 45, 4, 2002, 322-328.

[7] Parteli, E. J. R., Schmidt, J., Blumel, C., Wirth, K. E., Peukert, W. and Poschel, T., "Attractive Particle Interaction Forces and Packing Density of Fine Glass Powders," Scientific Reports, 4, 6227, 2014, $1-7$.

[8] Samadani, A. and Kudrolli, A., "Angle of Repose and Segregation in Cohesive Granular Matter," Physical Review E, 64, 2001, 051301.

[9] Cundall, P. A. and Strack, O. D. L., "A Discrete Numerical Model for Granular Assemblies," Geotechnique, 29, 1, 1979, 47-65.

[10] Lemieux, M., Leonard, G., Doucet, J., Leclaire, L. A., Viens, F., Chaouki, J. and Bertrand, F., "Large-Scale Numerical Investigation of Solids Mixing in a V-Blender Using the Discrete Element Method," Powder Technology, 181, 2, 2008, 205-216.

[11] Deen, N. G., Van Sint Annaland, M., van der Hoef, M. A. and Kuipers, J. A. M., "Review of Discrete Particle Modeling of Fluidized Beds," Chemical Engineering Science, 62, 1-2, 2007, 28-44.

[12] Feng, Y. T., Han, K. and Owen, D. R. J., “Discrete Element Simulation of the Dynamics of High Energy Planetary Ball Milling Processes," Materials Science and Engineering A, 375-377, 2004, 815819.

[13] Bierwisch, C., Kraft, T., Riedel, H. and Moseler, M., "ThreeDimensional Discrete Element Models for the Granular Statics and Dynamics of Powders in Cavity Filling," Journal of the Mechanics and Physics of Solids, 57, 1, 2009, 10-31.

[14] Tsunazawa, Y., Shigeto, Y., Tokoro, C. and Sakai, M., "Numerical Simulation of Industrial Die Filling Using the Discrete Element Method," Chemical Engineering Science, 138, 2015, 791-809.

[15] Thornton, C., "Numerical Simulations of Deviatoric Shear Deformation of Granular Media," Géotechnique, 50, 1, 2000, 43-53.

[16] Thornton, C. and Ning, Z., "A Theoretical Model for Stickbounce Behaviour of Adhesive, Elastic-Plastic Spheres," Powder Technology, 99, 2, 1998, 154-162.

[17] Thornton, C. and Yin, K. K., "Impact of Elastic Spheres with and without Adhesion," Powder Technology, 65, 1-3, 1991, 153-166.

[18] Renzo, A. D. and Maio, F. P. D., "Comparison of Contact-Force Models for the Simulation of Collisions in DEM-Based Granular Flow Codes," Chemical Engineering Science, 59, 3, 2004, 525-541.
[19] Akashi, M., Mio, H., Shimosaka, A., Shirakawa, Y., Hidaka, J. and Nomura, S., "Estimation of Bulk Density Distribution in Particle Charging Process Using Discrete Element Method Considering Particle Shape," ISIJ International, 48, 11, 2008, 1500-1506.

[20] Wu, Y., An, X. and Huang, F., "DEM Simulation on Packing Densification of Equal Spheres under Compression," Materials Research Innovations, 18, 2014, S4-1082-1086.

[21] Iwasawa, M., Tanikawa, A., Hosono, N., Nitadori, K., Muranushi, K. and Makino, J., "Implementation and Performance of FDPS: A Framework for Developing Parallel Particle Simulation Codes," Publications of the Astronomical Society of Japan, 68, 4, 2016, 54.

[22] Makino, J., "A Fast Parallel Treecode with GRAPE," Publications of the Astronomical Society of Japan, 56, 3, 2004, 521-532.

[23] Barnes, J. E. and Hut, P., "A Hierarchical O(N $\log N)$ ForceCalculation Algorithm," Nature, 324, 1986, 446-449.

[24] Barnes, J. E., “A Modified Tree Code: Don't Laugh; It Runs,” Journal of Computational Physics, 87, 1, 1990, 161-170.

[25] Le Van Sang, Yano, A., Fujii, S., Sugimura, N. and Washizu, H., "Coarse-Grained Model for Spring Friction Study of Micron-Scale Iron by Smoothed Particle Hydrodynamics," Europhysics Letters, $122,2,2018,26004-26009$.

[26] Le Van Sang, Yano, A., Isohashi, A., Sugimura, N. and Washizu, H., "Friction and Friction Heat of Micronscale Iron," Journal of Tribology, 142, 9, 2020, 091702-091707.

[27] Le Van Sang, Yano, A., Isohashi, A., Sugimura, N. and Washizu, H., "Smoothed Particle Hydrodynamics Study of Friction of the CoarseGrained $\alpha-\mathrm{Al}_{2} \mathrm{O}_{3} / \alpha-\mathrm{Al}_{2} \mathrm{O}_{3}$ and $\alpha-\mathrm{Fe}_{2} \mathrm{O}_{3} / \alpha-\mathrm{Fe}_{2} \mathrm{O}_{3}$ Contacts in Behavior of the Spring Interfacial Potential," Tribology International, 135, 2019, 296-304.

[28] Le Van Sang, Sugimura, N. and Washizu, H., "Influence of the Alumina Ceramic Coating on Friction and Stability of the Iron Contacts," Journal of Tribology, 143, 3, 2021, 031402.

[29] Le Van Sang, Yano, A., Osaka, A., Sugimura, N. and Washizu, H., "Adaptive Smoothed Particle Hydrodynamics for Study of Friction of Silica at Micronscale," Tribology Online, 15, 4, 2020, 259-264.

[30] Gunsteren, W. V. and Berendsen, H. J. C., "A Leap-Frog Algorithm for Stochastic Dynamics," Molecular Simulation, 1, 3, 1988, 173-185.

[31] Hertz, H., "On the Contact of Solid Elastic Bodies and on Hardness," Journal of Math, 92, 1881, 156-171.

[32] Thornton, C. and Liu, L., "DEM Simulations of Uni-Axial Compression and Decompression," Compaction of Solids, Granulates and Powders, CRC Press, 2000, 251-261.

[33] Landau, L. D. and Lifshitz, E. M., "Theory of Elasticity," Pergamon Press, New York, 1970.

[34] Ohmura, T., Zhang, L., Sekido, K. and Tsuzaki, K., “Effects of Lattice Defects on Indentation-Induced Plasticity Initiation Behavior in Metals," Journal of Materials Research, 27, 13, 2012, 1742-1749.

This paper is licensed under the Creative Commons Attribution-NonCommercial-NoDerivatives 4.0 International (CC BY-NC-ND 4.0) International License. This allows users to copy and distribute the paper, only upon conditions that (i) users do not copy or distribute such paper for commercial purposes, (ii) users do not change, modify or edit such paper in any way, (iii) users give appropriate credit (with a link to the formal publication through the relevant DOI (Digital Object Identifier)) and provide a link to this license, and (iv) users acknowledge and agree that users and their use of such paper are not connected with, or sponsored, endorsed, or granted official status by the Licensor (i.e. Japanese Society of Tribologists). To view this license, go to https://creativecommons.org/licenses/by-nc-nd/4.0/. Be noted that the third-party materials in this article are not included in the Creative Commons license, if indicated on the material's credit line. The users must obtain the permission of the copyright holder and use the third-party materials in accordance with the rule specified by the copyright holder. 